\title{
ON THE CLASSICAL LIMIT OF THE DIAGRAM EXPANSION IN QUANTUM STATISTICS
}

\author{
by T. W. J. M. JANSSEN \\ Instituut voor Theoretische Fysica, Rijksuniversiteit te Utrecht, Nederland
}

It is well known that the quantum statistical diagram expansions, as given for instance by Montroll and Ward ${ }^{1}$ ), by Lee and Yang ${ }^{2}$ ) and by Bloch and de. Dominicis ${ }^{3}$ ), bear a close resemblance to the classical Mayer cluster expansion ${ }^{4}$ ). An important difference is that in the quantum expansions the positions and also the temperatures of the various interactions differ, whereas in the classical expansion they are the same. In this note we want to demonstrate explicitly the way in which the quantum statistical diagram expansion, as given by Lee and Yang, in the classical limit $(h \rightarrow 0)$ tends to the Mayer diagram expansion.

The free particle propagator as defined by Lee and Yang is given by $g\left(\boldsymbol{r}^{\prime} \beta^{\prime} ; \boldsymbol{r} \beta\right) \begin{cases}\left\langle\boldsymbol{r}^{\prime}\left|\mathrm{e}^{-\left(\beta^{\prime}-\beta\right) \boldsymbol{H}_{0}}\right| \boldsymbol{r}\right\rangle=\left(\frac{m}{2 \pi\left(\beta^{\prime}-\beta\right) \hbar^{2}}\right)^{\prime} \exp \left[-\frac{m\left|\boldsymbol{r}-\boldsymbol{r}^{\prime}\right|^{2}}{2 \hbar^{2}\left(\beta^{\prime}-\beta\right)}\right] & \text { for } \beta^{\prime}>\beta \\ 0 & \text { for } \beta^{\prime}<\beta\end{cases}$

In the classical limit, when the thermal wave-length

one obtains

$$
\Lambda=\left(\frac{h^{2} \beta}{2 \pi m}\right)^{t} \rightarrow 0
$$

$$
g\left(\boldsymbol{r}^{\prime} \boldsymbol{\beta}^{\prime} ; \boldsymbol{r}, \beta\right) \rightarrow \delta\left(\boldsymbol{r}-\boldsymbol{r}^{\prime}\right) \quad \text { for } \quad \boldsymbol{\beta}^{\prime}>\beta
$$

i.e. the matrixelements of the unperturbed Boltzmann operator tend to $\delta$-functions.

Let us now consider the contribution of the diagram shown in fig. $1 a$

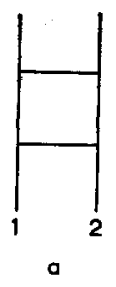

Fig. $1 a$

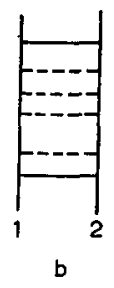

Fig. $1 b$

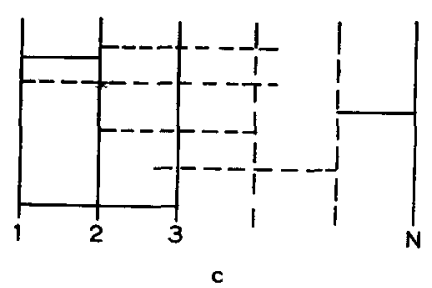

Fig. $1 c$ 
to the Slater sum $W_{2}$. It is given by

$$
\begin{aligned}
\int_{\beta>u_{1}>u_{2}} \mathrm{~d} u_{1} \mathrm{~d} u_{2} \int \mathrm{d} \boldsymbol{r}_{1}{ }^{\prime} \mathrm{d} \boldsymbol{r}_{2}{ }^{\prime} \mathrm{d} \boldsymbol{r}_{1}{ }^{\prime \prime} \mathrm{d} \boldsymbol{r}_{2}{ }^{\prime \prime} g\left(\boldsymbol{r}_{1} \beta ; \boldsymbol{r}_{1}{ }^{\prime} u_{1}\right) g\left(\boldsymbol{r}_{2} \beta ; \boldsymbol{r}_{2}{ }^{\prime} u_{1}\right) V\left(\boldsymbol{r}_{12}{ }^{\prime}\right) \times \\
\quad \times g\left(\boldsymbol{r}_{1}{ }^{\prime} u_{1} ; \boldsymbol{r}_{1}{ }^{\prime \prime} u_{2}\right) g\left(\boldsymbol{r}_{2}{ }^{\prime} u_{1} ; \boldsymbol{r}_{2}{ }^{\prime \prime} u_{2}\right) V\left(\boldsymbol{r}_{12}{ }^{\prime \prime}\right) g\left(\boldsymbol{r}_{1}{ }^{\prime \prime} u_{2} ; \boldsymbol{r}_{1} 0\right) g\left(\boldsymbol{r}_{2}{ }^{\prime \prime} u_{2} ; \boldsymbol{r}_{2} 0\right)
\end{aligned}
$$

and for $A \rightarrow 0$ its value tends to $\frac{1}{2} \beta^{2} A^{-6} V_{12}{ }^{2}$.

For the contribution of a ladder with $p$ interactions (cf. fig. $1 b$ ) one finds similarly in the limit

$$
\frac{(-1)^{p}}{p !} \beta^{p} \Lambda^{-6} V_{12}{ }^{p}
$$

From this one concludes that the contribution of a diagram with $N$ lines and $p$ interactions of which $m_{i j}$ between the pair $(i, j)$ (cf. fig. $1 c$ ) tends in the limit to

$$
\frac{(-1)^{p}}{p !} \beta^{p} \Lambda^{-3 N} \Pi_{i<j} V_{i j}^{m_{i j}}
$$

while the number of diagrams with the same contribution equals $p !\left(\Pi_{i<j} m_{i j} !\right)^{-1}$. On summing first the contributions of all diagrams with $N$ lines and $p$ interactions and then the contributions of all $N$-particle diagrams one obtains for the classical limit of the $N$-particle Slater sum

$$
W_{N}(1 \ldots N, 1 \ldots N) \equiv \sum_{n} \Psi_{n}{ }^{*}(1 \ldots N) \mathrm{e}^{-\beta H} \Psi_{n}(1 \ldots N)
$$

the value

$$
\begin{array}{r}
\lim _{A \rightarrow 0} W_{N}(1 \ldots N, 1 \ldots N)=\sum_{p=0}^{\infty} \frac{(-1)^{p}}{p !} \beta^{p} \Lambda^{-3 N} \sum_{\left\{m_{t i}\right\}}^{\prime} \Pi_{i<j} \frac{p ! V_{i j} m_{i j}}{m_{i j} !}= \\
=\Lambda^{-3 N} \mathrm{e}^{-\beta} \sum_{i<j} V_{i j}
\end{array}
$$

where $\Sigma^{\prime}$ indicates the sum over all distributions $\left\{m_{i j}\right\}$ such that $\sum_{i<j} m_{i j}=p$. Hence the limit of the canonical partition function becomes

$$
\begin{aligned}
\lim _{\Lambda \rightarrow 0} Z^{q} & =\lim _{\Lambda \rightarrow 0} \frac{1}{N !} \int \mathrm{d} \boldsymbol{r}_{1} \ldots \mathrm{d} \boldsymbol{r}_{N} W_{N}(1 \ldots N, 1 \ldots N) \\
& =\frac{1}{N !} \Lambda^{-3 N} \int \mathrm{d} \boldsymbol{r}_{1} \ldots \mathrm{d} \boldsymbol{r}_{N} \mathrm{e}^{-\beta V}=Z^{\bullet}
\end{aligned}
$$

where $Z^{q}$ and $Z^{c}$ denote the quantum and classical partition functions respectively, as was to be expected ${ }^{5}$ ).

From the foregoing it will be clear that a whole class of Lee-Yangdiagrams can be associated to one particular Mayer diagram, as is illustrated by way of example in the figs. $2 a$ and $2 b$.

The classical limit of the contributions from all the Lee-Yang diagrams indicated in fig. $2 a$ becomes, as we have shown

$$
\Sigma_{p=1}^{\infty} \frac{(-1)^{p}}{p !} \beta^{p} V_{12}{ }^{p} \Lambda^{-9}=\left(\mathrm{e}^{-\beta V_{12}}-1\right) \Lambda^{-9}=f_{12} \Lambda^{-9}
$$


i.e., apart from the factor $\Lambda^{-9}$, the contribution of the Mayer diagram shown in fig. $2 b$.

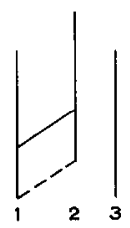

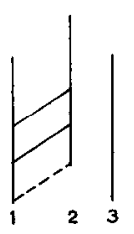

a

Fig. $2 a$

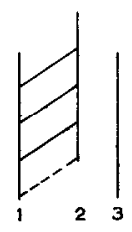

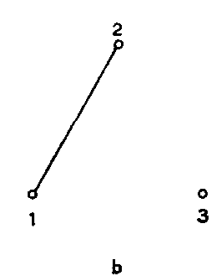

Fig. $2 b$

In a similar way one easily verifies that the connected Lee-Yang diagrams correspond to the connected Mayer diagrams. Two examples of this correspondence are schematically indicated in fig. 3 . As the well known clusterintegral $b_{l}$ both in quantum and classical statistics is given by integration of

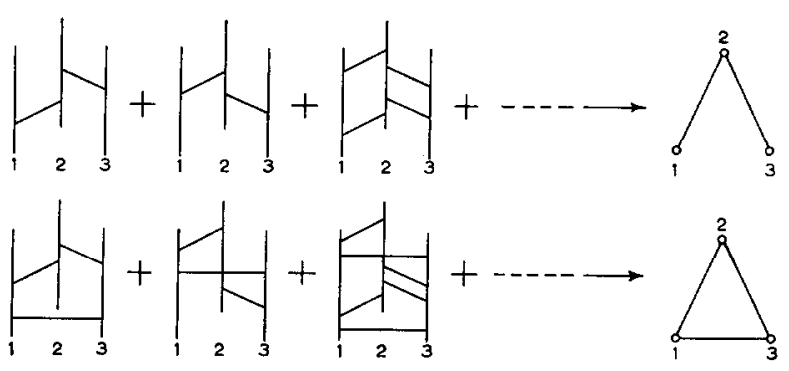

Fig. 3

the contributions of all connected diagrams of $l$ particles (in quantum statistics a factor $\Lambda^{3 l}$ must be included in the definition of $b_{l}$ ), it will be evident that

$$
\lim _{A \rightarrow 0} b_{l}^{q}=b_{l} c .
$$

I would like to thank prof. B. R. A. Nijboer for suggestions and advise concerning this note.

\section{REFERENCES}

1) Montroll, E. W. and Ward, J. C., Phys. of Fluids 1 (1958) 55.

2) Lee, T. D., and Yang, C. N., Phys. Rev. 113 (1959) 1165.

3) Bloch, C. and de Dominicis, C., Nuclear Phys. 10 (1959) 181.

4) Mayer, J. E. and Mayer, M. G., Statistical Mechanics, Wiley, New York 1940, Ch. XIII.

5) Kirkwood, J. G., Phys. Rev. 44 (1933) 31. 